\title{
La reflexión metapragmática como mecanismo del aprendizaje del imperativo en la clase de italiano como lengua extranjera
}

\author{
The metapragmatic reflection as a mechanism in learning the imperative form in the Italian class \\ as a foreign language
}

\author{
Elena A. Sánchez-Martínez ${ }^{a}$, Delphine Pluvinet ${ }^{b}$, Ma. De Lourdes Rico-Cruz ${ }^{c}$
}

\begin{abstract}
:
This paper presents the progress of a research work carried out with the data collected during the teaching of the imperative form to a group of students of an advanced course of Italian as a foreign language inside a Mexican public university in the center of the country. Throughout the data collection sessions, different activities were carried out that aimed to make students aware to some pragmatic aspects of the imperative in Italian, such as paralinguistic and kinetic elements. The partial results that are presented here, based on the analysis of the participants' discourse, show that the students' exposure to the various materials used, such as videos, audios and worksheets, where the pragmatics of the imperative is evinced, caused a reflection on the use of (im) politeness. This derives from the interpretation of the messages produced by the native speakers when they use the imperative, which developed a metapragmatic awareness in students. From these results, a didactic proposal for the teaching of cultural aspects is created where teachers adopt an important role in the teaching and learning processes of foreign languages in the classroom.
\end{abstract}

\section{Keywords:}

Metapragmatics, imperative, (im)politeness

\section{Resumen:}

En esta ponencia se presentan los avances de un trabajo de investigación que se realiza con los datos recolectados durante la enseñanza del imperativo a un grupo de estudiantes de un curso avanzado de italiano como lengua extranjera (ILE) de una universidad pública mexicana del centro del país. A lo largo de las sesiones de recolección de datos se realizaron actividades que pretendían sensibilizar a los estudiantes sobre algunos aspectos pragmáticos del imperativo en italiano, tales como elementos paralingüísticos y de la cinésica. Los resultados parciales que aquí presentamos, a partir del análisis del discurso de los participantes, muestran que la exposición de los estudiantes a los diversos materiales utilizados, como videos, audios y hojas de trabajo, donde se evidencia la pragmática del imperativo, provocó en ellos una reflexión sobre el uso de la (des)cortesía que se deriva de la interpretación de los mensajes producidos por los nativos cuando usan el imperativo, lo que a su vez generó en ellos el desarrollo de una conciencia metapragmática. A partir de estos resultados se genera nuestra propuesta didáctica de la enseñanza de los aspectos culturales, y que éstos adopten un rol importante dentro de los procesos de enseñanza y aprendizaje de lenguas extranjeras en las aulas.

\section{Palabras Clave:}

Metapragmática, imperativo, (des)cortesía

\section{INTRODUCCIÓN}

El aprendizaje de una lengua extranjera se basa en adquirir su gramática, su vocabulario, su sistema fonético, sus habilidades productivas y receptivas, así como las competencias necesarias para que un hablante de una lengua pueda convivir dentro del contexto de uso de la lengua extranjera sin obstáculos. Según el diccionario de términos clave de ELE (Centro Virtual Cervantes), de esto se encarga la pragmática, la cual estudia las relaciones que se dan entre el sistema de la lengua, por un lado,

\footnotetext{
a Correspondence Author, Universidad Autónoma de Querétaro, Facultad de Lenguas y Letras, https://orcid.org/0000-0001-8035-6127, Email: elena.andrea.sanchez@uaq.mx

b Universidad Autónoma de Querétaro, Facultad de Lenguas y Letras, https://orcid.org/0000-0001-7083-8691, Email: pluvinet.delphine@uaq.mx

c Universidad Autónoma de Querétaro, Facultad de Lenguas y Letras, https://orcid.org/0000-0001-9836-3886, Email: ricoc@uaq.mx
} 
y los interlocutores y el contexto de comunicación por otro. La pragmática forma parte explícita e implícita en la enseñanza dentro de las aulas de lengua extranjera. Su enseñanza, paralelamente a la de los contenidos gramaticales, fonéticos, culturales o de vocabulario, es esencial para lograr que los y las estudiantes aprendan a desenvolverse dentro de la lengua que están estudiando.

En el caso de esta investigación, se decidió observar el contexto en donde se aprende el imperativo en italiano, ya que cuando se aprende, causa cortes de comunicación en los estudiantes mexicanos, debido a que éste cuenta con distintos usos que en el español de México se pueden malinterpretar, haciendo que los y las aprendices mexicanos se sientan ofendidos ante el tono perentorio con el que los italianos utilizan este modo verbal.

En general, en español, se tiene la creencia que el imperativo se usa para ordenar. "La palabra imperativo deriva del latín imperare que significa "ordenar". En realidad no sólo sirve para esto, tiene otras funciones mucho más usadas en la comunicación cotidiana" (Bailini, 2005, p. 92). Cuando se empieza a enseñar el imperativo en italiano, los y las estudiantes mantienen la creencia implícita de que su uso, principalmente, es el de ordenar. Sin embargo, este modo verbal también cuenta con otros usos, y es cuando los y las aprendices se ven expuestos a éstos que empiezan a manifestar confusiones al recibir mensajes de los nativos. Éste es un problema meramente pragmático.

\section{APROXIMACIÓN TEÓRICA}

No fue hasta a finales del siglo XIX y comienzos del siglo XX que grandes exponentes de la Linguística como Chomsky llevaron a cabo los primeros estudios para trazar el camino que hoy conocemos como los estudios de Adquisición del Lenguaje desde una perspectiva lingüística. Para Vygotsky, por otro lado, el lenguaje tiene un origen social. "Evidentemente, el lenguaje desempeña algún papel en la comunicación de aspectos tanto cognitivos como socio-afectivos con los demás" (Mateo, 1982, p. 66). Así, Vygotsky destacó la importancia social del lenguaje y por consiguiente el fundamento social de su adquisición.

En este trabajo partimos de una perspectiva sociolingüística de la adquisición de segundas lenguas, donde el aprendizaje y uso de la lengua se ven como un proceso social ya que "consiste en entender el propio aprendizaje lingüístico como una acción socializadora: por ejemplo, la adquisición de la cortesía o de los usos propios de la conversación se fundamenta en la práctica de una acción social dentro de un contexto determinado" (Moreno, 2007, p. 56).

Una de las teorías sociolinguiísticas de adquisición de segundas lenguas, que tiene una gran relevancia dentro de este estudio, es la interaccionista. Éstas “intentan explicar la adquisición teniendo en cuenta que la interacción personal afecta a la percepción y la producción de la lengua adquirida y que, en su análisis, deben manejarse principios psico-socio-lingüísticos y pragmáticos" (Moreno, 2007, p. 59). Tomando como base esta teoría, se podría decir que, los malentendidos se producen debido a las diferencias en las normas que se encuentran inmersas en las culturas como lo son las clases sociales, las variaciones locales y el sexo de los hablantes; es por ello que es de suma importancia adecuar nuestros mensajes según la necesidad sociolingüística que tengamos (Hymes, 1995, citado en Betti, 2010). Por esta razón, la enseñanza de las manifestaciones socioculturales propias de una lengua, contextos donde aparecen, concientización y enseñanza de su pragmática, no pueden pasar a segundo plano o a un solo breve momento pedagógico dentro del aula de lengua, sino que "debe estar presente en toda la enseñanza y tratarse cada vez que los textos y los materiales didácticos utilizados ofrezcan una oportunidad o un pretexto" (Balboni, 2004 citado en Betti, 2010).

Sumándose a la importancia de lo anterior, según Macarena (2003), el ser humano se caracteriza por la capacidad de comunicase a través de distintos sistemas (gestual y escrito) y a través de signos vocales (lenguaje oral), según una modalidad híbrida que le permite expresarse de una forma más libre. Esto, sin duda, es un sistema más complejo que debe presuponer de parte de los docentes la importancia de su enseñanza y consideración dentro de sus planeaciones. Estos elementos forman parte de lo que se define como la comunicación no verbal, la cual consta, según Poyatos (2018), de emisiones de signos activos o pasivos, parte de un comportamiento, a través de los sistemas somáticos no léxicos, objetuales, espacios arquitectónicos, contenidos en una cultura concreta y producidos aisladamente o en mutua coestructuración.

La enseñanza de la comunicación no verbal de una lengua extranjera no debe obviarse ya que los elementos paralingüísticos, de acuerdo a Betti (2007), son igual de importantes como la palabra. Cuando hablamos de una personalidad propia de una lengua, podemos entenderla como la gama de componentes no verbales que acompañan cualquier lengua, o sea, el tono de voz y los gestos usados por los nativos de la lengua que se está estudiando. Al viajar al país extranjero de la lengua meta, los estudiantes podrían encontrar un bloqueo a la hora de comunicarse, y esto se debe a que "entre otras cosas, no tenemos competencia en el nivel paraverbal de esa lengua, es decir: la entonación, la ironía, las formas cómicas" (Betti, 2010, p. 18). Lo anterior se debe a que, en efecto, "la mayoría de la comunicación se da en un nivel no verbal: el cómo se dice algo es más importante que lo que se dice" (Argyle, 1978, citado en Betti, 2010, p. 19).

De acuerdo a Balboni (1998), las personas inmersas en una situación social de la lengua extranjera deben comprender el contexto en el que se mueven y usar palabras congruentes de acuerdo a la intensión del mensaje. Es importante entonces, conocer los códigos extralingüísticos, las reglas socioculturales, elegir el mensaje sociolingüístico adecuado, así como los elementos paralingüísticos y pragmáticas correctos. Aquí es donde el proceso de la obtención de una conciencia metapragmática toma fuerza, ya que según Verschueren (2000), ésta es el conocimiento del significado social de las distintas variables de una segunda lengua y de cómo aparecen éstas en 
distintos contextos. Podríamos entonces entenderla como la competencia que una persona posee para comunicarse eficazmente dentro de distintas situaciones en un ambiente de la lengua estudiada.

Finalmente, al ver que los usos pragmáticos del imperativo en español y en italiano son distintos y crean mensajes que se interpretan como descorteses, se decidió insertar dentro de este estudio el tema de la cortesía. La importancia de la cortesía en las clases de lengua se reconoce, como Haugh \& Kádár (2013) comentan, como un medio clave a través del cual los humanos resuelven y mantienen relaciones interpersonales. La cortesía cubre algo mucho más amplio, abarcando todo tipo de comportamiento interpersonal a través del cual tomamos en cuenta los sentimientos de los demás sobre cómo piensan que deberían ser tratados en el ejercicio y el mantenimiento de nuestro sentido de la personalidad, así como nuestras relaciones interpersonales con los demás.

Lo anterior respalda el camino a seguir en una clase de lengua extranjera que aspire a formar a los estudiantes tanto desde el punto de vista lingüístico como en el aspecto cultural. Basándonos en Garrido (2005), el estudiante debe adquirir habilidades comunicativas y sociales desde el primer momento de su aprendizaje y dichas habilidades deben estar estrechamente relacionadas con la cortesía. Si tomáramos en cuenta que al ser maestros de lengua lo somos en automático de cultura, entenderíamos inmediatamente el gran compromiso de enseñar las competencias sociolingüísticas a nuestros estudiantes.

\section{METODOLOGÍA}

El tema que nos compete en esta investigación es el análisis de las reflexiones metapragmáticas realizadas por las y los estudiantes de italiano como lengua extranjera durante la enseñanza del imperativo. Para analizar las reflexiones realizadas por los y las participantes, fue necesario el análisis de una narrativa donde se vio evidenciado el discurso usado por los mismos así como las reflexiones metapragmáticas a las que llegaron. Al ser un estudio con enfoque cualitativo, éste tiene características sociales, ya que "en esta perspectiva, el investigador es parte del mundo social que estudia" Aravena, Kimelman, Micheli, Torrealba \& Zúñiga, 2006, p. 39).

Posterior a las sesiones realizadas para la recolección de datos, se decidió que el proceso que se usaría para el análisis de datos de este estudio sería una adaptación del propuesto por Sampieri (Hernández, 2004). El diseño que se hizo especialmente para este estudio consta de los siguientes pasos:

- Paso uno: revisión del material obtenido en la recolección de datos.

- Paso dos: elaboración de un plan de trabajo donde se evidenciaron los pasos a seguir en el análisis de datos.

- Paso tres: codificación de los datos de la narrativa obtenida a través de la entrevista al grupo focal.

- Paso cuatro: interpretación de los datos.
- Paso cinco: identificación de patrones dentro de la narrativa, se explicaron sucesos y se construyó una teoría.

- Paso seis: revisión del estudio a dos expertos del área de italiano para asegurar la confiabilidad y dar validez al estudio.

- Paso siete: retroalimentación del estudio y se elaboraron las correcciones necesarias para repetir el estudio en caso de ser necesario.

Asimismo, se decidió analizar la narrativa recolectada a través de una entrevista semiestructurada que se hizo a un grupo focal donde se evidenciaron las reflexiones metapragmáticas realizadas por los y las participantes. Una vez realizada la recolección de datos, se revisó la información obtenida a través de dicha entrevista, se clasificó y se analizó. Aravena et al. (2006) comentan que la entrevista es una herramienta particularmente útil para los investigadores sociales ya que permite acceder al conocimiento de la vida social a través de relatos verbales. Posterior a la entrevista, se analizaron los datos obtenidos de éstas a través de las transcripciones realizadas de la entrevista semiestructurada llevada a cabo con el grupo focal de los y las participantes.

Para explicar lo hallado y para la interpretación de los datos, se tomó en cuenta lo siguiente para la creación de categorías: a) descripción, b) definición y c) frecuencia de aparición de palabras, y se procedió a encontrar patrones que llevaran a entender los fenómenos ocurridos en el estudio. Para ello se describieron los participantes, sus contextos, el contexto donde se llevó a cabo el estudio, sus actores, los materiales usados para la fase de sensibilización del tema y las sesiones, las herramientas de recolección de datos, los instrumentos y las etapas de análisis de datos.

\section{Diseño metodológico}

El diseño metodológico de la investigación del tema se llevó a cabo en seis pasos. El "Paso 1: Planeación y selección de materiales", "Paso 2: Diseño de secuencia didáctica", "Paso 3: Selección de participantes y pilotaje", "Paso 4: Revisión cambios de pilotaje", "Paso 5: Planeación de estudio principal", "Paso 6: Puesta en práctica del estudio principal". Posteriormente, se firmó el consentimiento de participación, se llenó una encuesta respecto a su contexto académico y personal. Después se llevaron a cabo las sesiones, las cuales se dividieron en tres con una duración de dos horas cada una. Por último, se hizo una entrevista al grupo focal.

\section{Instrumentos de recolección}

Dentro del marco de la investigación, se usaron los siguientes instrumentos para recolectar datos.

- Hojas de trabajo: en estas hojas se tenía que responder a ejercicios escritos en imperativo.

- Evaluación final escrita y oral: al final de las actividades planeadas y una vez generada una conciencia pragmática, los estudiantes contestaron a 
una evaluación la cual constaba de preguntas sociopragmáticas sobre el uso del imperativo.

- Entrevista semiestructurada al grupo focal: ésta se realizó con el fin de generar una reflexión metapragmática sobre el uso del imperativo.

\section{Herramientas}

Dentro del marco de la investigación, se usaron las siguientes herramientas de forma complementaria a los instrumentos de recolección de datos.

- Encuesta: con el fin de conocer los antecedentes académicos y contexto personal de los y las participantes, así como el conocimiento del imperativo y su pragmática y nivel de lengua.

- Secuencia didáctica: la planeación de la secuencia didáctica contenía las siguientes actividades: a) Audio de diálogo en imperativo, b) Mini diálogos en imperativo, c) Blogs italianos, d) Extractos de una película.

\section{Contexto}

El estudio se llevó a cabo dentro de los cursos curriculares de italiano en un centro de lengua de una universidad pública de México. Los cursos tienen una duración de ocho semestres, los estudiantes terminan con un nivel B2 o C1 según el MCER* Cada nivel está compuesto de 90 horas de clases por semestre. Dentro de los cursos se encuentran personas de 15 hasta 80 años.

\section{Participantes}

El total de los y las participantes en la recolección de datos fue de cuatro, de edad comprendida entre los 22 y 27 años, pertenecientes a las Facultades de Lenguas y Letras y Bellas Artes de la misma universidad donde se llevó a cabo la investigación. Todos son hablantes nativos del español; además del italiano conocen en diferentes niveles otras lenguas como el inglés y el francés. Los estudiantes tienen un nivel de italiano B2 de acuerdo al MCER. Durante la investigación, cursaban el séptimo semestre de italiano de ocho en total, lo cual es equivalente a 630 horas de estudio de la lengua italiana.

\section{Etapas de análisis cualitativo del discurso narrativo}

Esta etapa se empezó con la organización de la información. Los datos obtenidos se codificaron para tener la información más resumida y poder encontrar categorías temáticas de la investigación en los extractos más significativos. La transcripción de la entrevista se hizo en un documento con cuatro columnas, donde se puso el número de intervención, el minuto donde se interviene, la transcripción codificada y los comentarios con las categorías encontradas. Para poder encontrar las categorías que dieran significado a las intervenciones de los y las participantes en la entrevista, la docente investigadora tuvo que "hacerse preguntas tales como: ¿Qué tópico o tópicos aparecen en la mayoría de los grupos?,
¿Existen diferentes opiniones respecto a dichos tópicos?, ¿De qué factores depende la variación de las opiniones?" (Aravena et al, 2006: 90).

\section{RESULTADOS}

Los resultados parciales obtenidos hasta este momento a través del estudio principal mostraron en primera instancia que existiría una tendencia a desarrollar una conciencia metapragmática a través de las actividades que los estudiantes sostuvieron sobre el imperativo y sus usos pragmáticos en la lengua italiana.

A través del análisis del discurso de la entrevista, se crearon dos principales categorías: reflexión metalingüística y reflexión metapragmática. A su vez, dentro de las dos categorías principales, se crearon sub-categorías para explicar los fenómenos que aparecieron durante el análisis de la entrevista. Dentro de la categoría "reflexión metalingüística" se establecieron las subcategorías "reflexión metacognitiva" y "reflexión comparativa entre la L1 y la L2"; mientras que en la categoría "reflexión metapragmática" se designó la subcategoría "reflexión de sistemas paralingüísticos y sistema cinésico". En esta investigación sólo nos enfocaremos en discutir la última categoría, debido a que es lo que más apareció en el análisis y debido a que el fenómeno de la pragmática es un área de investigación que promete grandes aportaciones dentro de los procesos de enseñanza y aprendizaje de lenguas extranjeras. Esto se pudo observar en la entrevista final en donde se hizo una reflexión metapragmática por parte de los estudiantes, ya que durante las preguntas y respuestas que se daban, apareció una reflexión treinta y tres ocasiones elaborada ellos mismos. Esto representa un total del $38 \%$ del total del discurso analizado. Para una mayor comprensión, se incluyen algunos ejemplos obtenidos durante la entrevista.

A continuación se muestra el código usado para identificar la aparición de frases o palabras pertenecientes a las siguientes categorías:

1. ++ Reflexión metalingüística ++

a. $\quad\{\{$ Reflexión metacognitiva $\}\}$

b. ::Reflexión comparativa entre L1 y L2::

2. ${ }^{\circ}$ Reflexión metapragmática ${ }^{\circ}$

a. ר Comunicación no verbal (uso del sistema paralingüístico y sistema cinésico) ר

P: ¿Cómo podrían describir a los italianos, en comparación a los mexicanos, en el uso del imperativo?

S3: ${ }^{\circ}$ Rudos ${ }^{\circ \circ}$

S1: $\quad$ LYo pienso que ${ }^{\circ}$ los italianos son ${ }^{\circ}$, en ese contexto, ${ }^{\circ}$ como que quieren llamar más la atención ${ }^{\circ \circ}$, o sea como que ${ }^{\circ}$ su tono es más para llamar más la atención ${ }^{\circ}$, independientemente del uso, como que siempre es $\neg$ truena los dedos רמר "pero ponme atención, o sea te estoy dando esta orden y ponme atención". Y nosotros como que "ah" ᄀרusa voz floja, reflejando la nula voluntad de hacer algo רמל “haz esto!”. A lo

* El Marco Común Europeo de Referencia, por sus siglas MCER de ahora en adelante, es un proyecto que se inscribe dentro de la política lingüística del Consejo de Europa. 
mejor es porque a veces ${ }^{\circ}$ estamos acostumbrados a que "me lo va a decir otra vez"००, por ejemplo las mamás, ¿no? Cuando te dicen "¡lava los trastes!” y "ah sí, ahorita”. "¡QUE LAVES LOS TRASTES!” ᄀרusa voz agresiva alzando la voz ר y conforme más te lo van diciendo, más te van subiendo el tono. ${ }^{\circ}$ Ya cuando ves con la chancla *risas* ya es otro show ${ }^{\circ \circ}$

S2: Pues yo digo que ${ }^{\circ \circ}$ son muy expresivos ${ }^{\circ \circ}$, como había dicho Luz, ${ }^{\circ}$ con los gestos y las manos ${ }^{\circ \circ}$. Se les escucha su tonito, como muy cantadito. Luego ${ }^{\circ}$ nosotros no le ponemos tanto énfasis ${ }^{\circ \circ}$ a las cosas que decimos, solo decimos plano y ellos sí como que le ponen mucho énfasis

S1: $L_{\text {mucha crema a sus tacos }}$

S2: Ajá

P: ¿Y ese énfasis cómo es? ¿Es fuerte, es bonito, es negativo, es feliz?

S2: Sí es ${ }^{\circ}$ fuerte ${ }^{\circ \circ}$, ¿no? $\neg$ Suben el tono de voz $ר$

S3: Y también puede ser ${ }^{\circ \circ}$ un poco negativo ${ }^{\circ \circ}$

S1: Depende de lo que te estén diciendo

P: Depende... Pero normalmente sí *risas*

S4: Como que dan ${ }^{\circ}$ un poco de miedo ${ }^{\circ \circ}$

S2: Y hablan muy rápido también, ¿o es sólo mi impresión?

En este ejemplo se pueden observar las distintas reflexiones metapragmáticas realizadas por las estudiantes donde, incluso, hicieron uso del sistema paralingüístico y cinésico. Mientras, la participante S1 compara las tonalidades de voz que se usan en italiano para dar énfasis a las palabras y su uso en español, las participantes S2, S3 y S4 reflexionan nuevamente sobre cómo el tono de voz afecta a la percepción de los mensajes en italiano: ellas lo perciben de una forma fuerte, negativa e incluso les causa un poco de miedo.

En la figura 1 se pueden muestran los porcentajes de las palabras o frases que encajan en cada una de las categorías y subcategorías ligadas a esta investigación. Claramente se puede ver que el mayor porcentaje es el de la reflexión metapragmática realizada por los estudiantes. Analizar este fenómeno fue muy relevante ya que cualquier docente de lenguas extranjeras conoce la importancia de la enseñanza de los temas relacionados con la cultura de los países de la L2, la pragmática es una parte esencial de la lengua que se ha observado que no es tomada en consideración en las planeaciones de clase.

Figura 1: Frecuencia de aparición de frases y palabras ligadas a las categorías del análisis del discurso de la entrevista final al grupo focal.



FUENTE: Elaboración propia

Es importante aclarar que los resultados obtenidos hasta el momento son parciales. No se puede aún generalizar, ya que este estudio tiene muchas limitaciones; además, el proceso de análisis de resultados aún está en curso. Sin embargo, en los estudiantes se puede ver la tendencia al desarrollo de una conciencia metapragmática debido a un estudio del imperativo y sus usos donde la pragmática juega un rol importante.

\section{CONCLUSIONES}

En este artículo se ha reflexionado sobre la importancia de la pragmática en la enseñanza y aprendizaje de una lengua extranjera, así como la importancia del fenómeno de la cortesía y el rol que juega dentro del imperativo en italiano, así como por qué la enseñanza de la cultura propia de la L2 debe formar parte de los contenidos de un curso de lengua.

Los primeros análisis del estudio en curso, nos han permitido observar, que los estudiantes, al finalizar el mismo, realizaron una reflexión metapragmática sobre los usos del imperativo en italiano; una reflexión metalingüística sobre las diferencias existentes en el tema entre su lengua materna (el español) y la lengua meta $\mathrm{y}$, finalmente, una reflexión metacognitiva al reflexionar sobre las formas que ellos usan para aprender la L2. Debido a que en esta primera etapa de la investigación, nos centramos solo en la reflexión metapragmática de los estudiantes, al respecto podemos decir que las reflexiones metapragmáticas que los estudiantes generaron dentro de este estudio fueron bien recibidas por ellos e influyeron de manera positiva en sus percepciones tanto de la lengua materna como de la lengua extranjera, sus culturas y sus aprendizajes. El favorecer esta conciencia estimula que los estudiantes generen una mayor conciencia y entendimiento de los contextos culturales de la lengua extranjera en los cuales se desenvuelven los estudiantes y, al mismo tiempo, que se beneficien de una mejor y más eficaz apropiación de los contenidos gramaticales, sintácticos, fonéticos, morfológicos y culturales, logrando el objetivo de convertirse en agentes sociales dentro de la lengua extranjera.

\section{REFERENCES}


Aravena, et al. (2006). Investigación Educativa I. Universidad Arcis, Chile. Recuperado

http://jrvargas.files.wordpress.com/2009/11/investigacion-

educativa.pdf, fecha de consulta 14 de octubre de 2019.

Betti, Silvia (2010). «Análisis contrastivo y cortesía: “¿El cómo se dice algo es más importante que lo que se dice?"» Revista de Lenguas para Fines Específicos 15/16: 13-38.

Betti, Silvia (2001). «Comunicación no verbal y gestualidad: "El cómo se dice algo es más importante que lo que se dice" Estudio contrastivo entre español e italiano».

Centro Virtual Cervantes. «Pragmática». En Diccionario de términos clave de ELE. Accedido 14 de octubre de 2019. https://cvc.cervantes.es/ensenanza/biblioteca_ele/diccio_ele/diccionari o/pragmatica.htm.

Cestero, Ana Ma. «La Comunicación no verbal: propuestas metodológicas para su estudio». Lingüística en la red, 2016, 1-36.

Fernández, L (2006). ¿Cómo analizar datos cualitativos? Butlletí La Recerca. Universitat de Barcelona.

Garrido, Ma del Camino. «Cortesía y actos de habla en la enseñanza de E/LE». En La competencia pragmática y la enseñanza del español como lengua extranjera., 308-17. Oviedo, España: Universidad de Oviedo, 2006

Haugh, M. y Dániel Kádár (2013). «Introduction». En Understanding Politeness, 1st edition. 1-13. Cambridge, UK: Cambridge University Press. Recuperado de http://real.mtak.hu/23508/1/HaughKadar2013_Understandings_of_Poli teness_English_translation-libre\%20copy.pdf, fecha de consulta 14 de octubre de 2019 .

Mateo, F. A., \& Sagastume, F. E. (1982). Contexto sociocultural y adquisición del lenguaje. Kobie, 3, 63-72. Recuperado de https://cutt.ly/ted1K7h, fecha de consulta 14 de octubre de 2019.

Poyatos, Fernando. «Los estudios de comunicación no verbal como rama interdisciplinar de la lingüística». Lingüística en la red, 2018, 1-31.

Verschueren, Jef. «NOTES ON THE ROLE OF METAPRAGMATIC AWARENESS IN LANGUAGE USE». International Pragmatics Association 10, n. ${ }^{\circ}$ (2000): 439-56. 